\title{
Autologous haematopoietic stem cell transplantation (aHSCT) for severe resistant autoimmune and inflammatory diseases - a guide for the generalist
}

\author{
Authors: John A Snowden, ${ }^{\mathrm{A}}$ Basil Sharrack, ${ }^{\mathrm{B}}$ Mohammed Akil, ${ }^{\mathrm{C}}$ David G Kiely, ${ }^{\mathrm{D}}$ Alan Lobo, ${ }^{\mathrm{E}}$ Majid Kazmi, \\ Paolo A Muraro ${ }^{G}$ and James $O$ Lindsay ${ }^{H}$
}

Autologous haematopoietic stem cell transplantation (aHSCT) is commonly used for the treatment of haematological cancers, but is increasingly used in the treatment of patients severely affected by autoimmune diseases (ADs). In fact, ADs have become the fastest growing indication for aHSCT. A wide range of diseases have been treated, but the field has focused on three areas: multiple sclerosis, diffuse cutaneous systemic sclerosis and Crohn's disease, where there are populations of patients for whom disease control remains unsatisfactory despite the advent of biological and targeted small molecule therapies. Scientific studies of immune reconstitution have provided support for a 'rebooting' of the immune system through a re-diversification of naive and regulatory immune effector cells. In addition, there may be health economic benefits from a single one-off procedure. Even so, the treatment with aHSCT is intensive with a range of toxicities and risks which, despite being routine to transplant haematologists, are less familiar to disease specialists. Close collaboration between transplant haematologists and relevant disease specialists in patient selection, clinical management and follow-up is mandatory. Ideally, patients should be treated on a clinical trial if available.

Authors: ${ }^{\mathrm{A}}$ consultant haematologist, director of BMT and chair of the Autoimmune Diseases Working Party (ADWP) of the European Society for Blood and Marrow Transplantation (EBMT), Department of Haematology, Sheffield Teaching Hospitals NHS Foundation Trust, Royal Hallamshire Hospital, Sheffield, UK; ${ }^{B}$ consultant neurologist, Department of Neurology, Sheffield Teaching Hospitals NHS Foundation Trust, Royal Hallamshire Hospital, Sheffield, UK; ' Consultant rheumatologist, Department of Rheumatology, Sheffield Teaching Hospitals NHS Foundation Trust, Royal Hallamshire Hospital, Sheffield, UK; ${ }^{\text {D consultant }}$ respiratory physician, Sheffield Pulmonary Vascular Disease Unit, Sheffield Teaching Hospitals NHS Foundation Trust, Royal Hallamshire Hospital, Sheffield, UK; ${ }^{{ }^{E}}$ consultant gastroenterologist, Department of Gastroenterology, Sheffield Teaching Hospitals NHS Foundation Trust, Royal Hallamshire Hospital, Sheffield, UK; ' F consultant haematologist, Kings Healthcare Partners, London, UK; ${ }^{G}$ professor of neurology, neuroimmunology and immunotherapy, Brain Sciences, Imperial College London, London, UK; ${ }^{H}$ professor of inflammatory bowel disease, Centre for Immunobiology, Barts and the London School of Medicine and Dentistry, Blizard Institute, Queen Mary University of London, London, UK

\section{Introduction}

Haematopoietic stem cell transplantation (HSCT) has evolved over the past 50 years to treat a wide range of conditions and is now a routine part of clinical haematological practice (Table 1). Autologous HSCT (aHSCT) is widely used to treat malignant diseases such as myeloma and lymphoma. With time, aHSCT has become safer and its use has also extended to non-malignant diseases, including severe autoimmune diseases (ADs), which is now the fastest growing indication for $\mathrm{HSCT}^{1}{ }^{1}$

Clinical studies of aHSCT in ADs have been ongoing since the mid-1990s. However, it has taken two decades for aHSCT to be

\section{Key points}

Autologous HSCT has the potential to induce sustained clinical remissions in otherwise resistant or poor prognosis autoimmune diseases enabling reduction and even long-term withdrawal of immunosuppressive and biological therapies

Although a wide range of autoimmune diseases have been treated, the evidence base is greatest in relapsing remitting MS, diffuse systemic sclerosis and Crohn's disease

Autologous HSCT requires close collaboration between experienced transplant haematologists and relevant disease specialists in patient selection, management and followup. Early referral of patients is essential to optimise clinical outcomes and minimise risks

Autologous HSCT may lead to 'rebooting' of the immune system through regeneration and rediversification of the $T$ and B-cell repertoire and increased regulatory $\mathrm{T}$-cell activity

There may be health economic benefits to a one-off intensive treatment over long-term administration of biological and immunosuppressive therapy

KEYWORDS: Autoimmune diseases, haematopoietic stem cell transplantation, stem cells, autologous, blood and marrow transplantation 
Table 1. Glossary of terms used in haematopoietic stem cell transplantation (HSCT)

\section{Types of HSCT}

Autologous HSCT (aHSCT)

Transplantation of haematopoietic stem cells taken from patients and reinfused after high doses of cytotoxic therapy (usually chemotherapy with immunoablative therapeutic antibodies such as anti-thymocyte globulin [ATG] in patients with severe autoimmune diseases)

Allogeneic Transplantation of haematopoietic stem cells HSCT from a donor, either sibling or unrelated, and occasionally cord blood. Allogeneic HSCT is rarely performed for autoimmune diseases given the higher risks, and its use has been largely restricted to paediatrics

\section{Sources of haematopoietic stem cells (HSC)}

Peripheral blood stem cells

Bone marrow

\section{HSCT phases}

Mobilisation

Conditioning

Harvesting

'Transplant'/ reinfusion

Aplastic phase

Supportive care

The combination of antibiotic (prophylactic

Engraftment and therapeutic), transfusions, symptomatic care, growth factors and monitoring required to bring the patient safely through and beyond engraftment, usually around 2 weeks posttransplantation

Mobilisation of CD34+ stem cells from the bone marrow into the peripheral blood using granulocyte colony stimulating factor (G-CSF) +/chemotherapy. Stem cells are then harvested using leukapheresis

High dose immunoablative or immunosuppressive regimen usually chemotherapy and therapuetic antibodies (for example antithymocyte globulin [ATG]) administered prior to stem cell infusion Stem cells are harvested using leukapheresis

The thawing and reinfusion of the $\mathrm{CD} 34+$ cells into the patient

The period when the immune system and haematopoietic system are unable to produce sufficient cells to maintain blood counts and innate immunity. The combination of antibiotic (prophylactic and therapeutic), transfusions, symptomatic care, growth factors and close monitoring required to bring the patient safely through and beyond engraftment, usually around 2 weeks post-transplantation

Defined as 3 days of neutrophils rising above $0.5 \times 10^{9} / \mathrm{L}$ and/or platelets rising above $20 \times 10^{9} / \mathrm{L}$ unsupported recognised as a treatment option among the evolving array of biologic and other modern therapies. More recent evidence has supported its use in well-selected patients with ADs, especially multiple sclerosis (MS), systemic sclerosis (SSC) and Crohn's disease (CD). ${ }^{2,3}$ Consensus recommendations have been published to guide judicious patient selection and multidisciplinary practice of aHSCT across a range of ADs. ${ }^{4-8}$

\section{Multiple sclerosis}

Current disease modifying therapies (DMTs) reduce relapse rates in patients with relapsing remitting MS (RRMS) but their impact on disability is limited. DMTs for progressive MS are scarcely effective and early intervention at the inflammatory RRMS phase of this illness is therefore key to preventing long-term disability. ${ }^{9-11}$

Autologous haematopoietic stem cell transplantation has been used in the treatment of MS since 1995. Initially, patients with advanced progressive disease were treated with limited benefit. ${ }^{9-11}$ A small phase II randomised controlled trial (RCT) confirmed reduced magnetic resonance imaging (MRI) disease activity with aHSCT, ${ }^{12}$ which strongly supported further phase III studies with primary clinical endpoints. There is now increasing evidence that its early use in patients with highly active RRMS results in significant reduction in clinical and MRI disease activity as well as improving patients' disability to a significantly greater degree compared with currently available DMTs. ${ }^{9-11}$ The recent results of the MIST study (ClinicalTrials.gov identifier: NCT00273364), which compared the effectiveness of aHSCT against standard DMTs, suggests that aHSCT can be safely administered and is significantly more effective. ${ }^{13}$ However, alemtuzumab, one of the most effective DMTs, was not included in the control arm. An RCT of aHSCT with alemtuzumab would resolve the question of relative efficacy.

Outside of clinical trials, current consensus supports the use of aHSCT in patients with RRMS who are young ( $<45$ years old), able to ambulate independently, have illness duration of $<10$ years, and at least two clinical relapses in the previous year despite the use of DMTs with MRI evidence of concurrent disease activity. Patients with higher degrees of disability, or occasionally progressive disease course, can be considered if there is clear evidence of significant clinical and MRI disease activity although the benefit in these patients is more limited. Potential patients should be discussed with, and treated in, specialised centres with an interest in aHSCT for MS.

\section{Rheumatological diseases}

SSc is a multisystem autoimmune connective tissue disease; rapidly progressive diffuse cutaneous SSc (dcSSc) has a 5-year mortality up to $30 \%$. Vasculopathy, excessive collagen deposition, inflammation in the skin and internal organs (lung, gastrointestinal, cardiovascular and renal) result in fibrosis and organ failure, with death most commonly a consequence of pulmonary arterial hypertension or interstitial lung disease. Effective drug therapy for SSc is lacking; disease-modifying antirheumatic drugs (DMARDs) and biologics have failed to show lasting benefit; cyclophosphamide over 12 months has shown short-term benefit compared with placebo. ${ }^{4,8,14-16}$

Three RCTs in SSc comparing aHSCT to cyclophosphamide have been reported: ASSIST, ${ }^{14} \mathrm{ASTIS}^{15}$ and SCOT ${ }^{16}$ (Table 2). All studies compared aHSCT to monthly pulsed intravenous 
Table 2. Registrations in the EBMT Registry for

autologous HSCT in autoimmune diseases $(n=2515)$, June 2018*

Multiple sclerosis

Connective tissue

Systemic sclerosis

SLE

Polymyositis-dermatomyositis

Sjogren's syndrome

4

Antiphospholipid syndrome

6

Other/unknown

\section{Arthritis}

Rheumatoid arthritis

Juvenile chronic arthritis:

Systemic JIA

Other JIA

Polyarticular JIA

Psoriatic arthritis

Other

Inflammatory bowel disease

Crohn's disease

Ulcerative colitis

Other

Haematological

Immune thrombocytopenia

Evans' syndrome

Autoimmune haemolytic anaemia

Other

\section{Vasculitis}

4

Wegener's type

Behcet's disease

Takayasu

Polyarteritis

Churg-Strauss

HUVS

Other/unknown

\section{Other neurological}

Neuromyelitis optica

CIDP

Myasthenia gravis

Other/unknown

*EBMT registry accessed 4 June 2018.

CIDP = Chronic inflammatory demyelinating polyneuropathy ; HUVS = hypocomplementemic urticarial vasculitis syndrome; JIA = juvenile idiopathic arthritis cyclophosphamide. ASSIST randomised patients to aHSCT or cyclophosphamide for 6 months and 19 patients were followed for 2 years. There was a significant improvement in respiratory and cutaneous disease in the aHSCT group with no transplant related mortality (TRM) reported. ASTIS compared aHSCT to cyclophosphamide for 12 months in 156 patients with early dcSSc and a median follow-up of 5.8 years. The primary endpoint was event-free survival (time from randomisation until the death or persistent major organ failure). In the aHSCT arm there were 22 events including 19 deaths (TRM 10\%) predominantly attributed to SSc-related cardiac dysfunction. Long-term event-free and overall survival rates were higher with aHSCT. SCOT randomised 75 patients with severe SSc to aHSCT versus cyclophosphamide for 12 months, followed up for 4.5 years. The primary endpoint was a global rank composite score (GRCS) based on a hierarchy of disease features. AHSCT resulted in significantly better clinical outcomes compared with cyclophosphamide, with fewer events of conditioning-associated cardiac toxicity and TRM was reduced compared with ASTIS.

Improvements in disease control and long-term survival make aHSCT in selected patients with SSc increasingly attractive. Given that pre-existing pulmonary vascular and cardiac involvement are major contributors to TRM risk, an extensive cardiothoracic evaluation is mandatory for appropriate patient selection. ${ }^{5}$

AHSCT has also been used successfully in the treatment of other severe autoimmune connective tissue diseases including systemic lupus erythematosus (SLE), ${ }^{17}$ rheumatoid and other inflammatory arthritides, dermato- and polymyositis and systemic vasculitis. ${ }^{3,4}$ However, in contrast to SSC, the success of standard immunomodulatory treatments and newer biological therapies in the management of these diseases has been such that in practice aHSCT is only considered for those individuals with severe recalcitrant disease.

\section{Crohn's disease}

Despite advances in conventional and biological therapies, a substantial minority of $C D$ patients experience treatment refractory disease that results in a marked reduction in quality of life, leaving such individuals with limited therapeutic options. Surgical resection may not be feasible or may be declined by the patient if it will result in a permanent stoma. ${ }^{6}$

Initial case series and single-centre cohort studies suggested that aHSCT could induce sustained clinical benefit. To date there has only been one randomised controlled trial to assess the value of aHSCT in refractory CD, the ASTIC trial $^{18}$ (see Table 3). ASTIC was a negative trial partly due to a very ambitious primary endpoint, and partly as the control arm also delivered a significant dose of cyclophosphamide for stem cell mobilisation resulting in transient reduction in clinical disease activity. Patients randomised to the control group in ASTIC could undergo aHSCT after the primary endpoint had been assessed and underwent the same schedule of assessments over the subsequent year. When analysed as the combined cohort of all 40 patients that underwent aHSCT, there were significant improvements in clinical disease activity, quality of life and endoscopic disease activity after one year; $50 \%$ of this group had complete mucosal healing, an important endpoint in CD and associated with improved longterm outcomes. ${ }^{19}$

Long-term outcomes after aHSCT are also reported in singlecentre and registry cohorts. Significantly, the majority of 


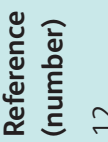

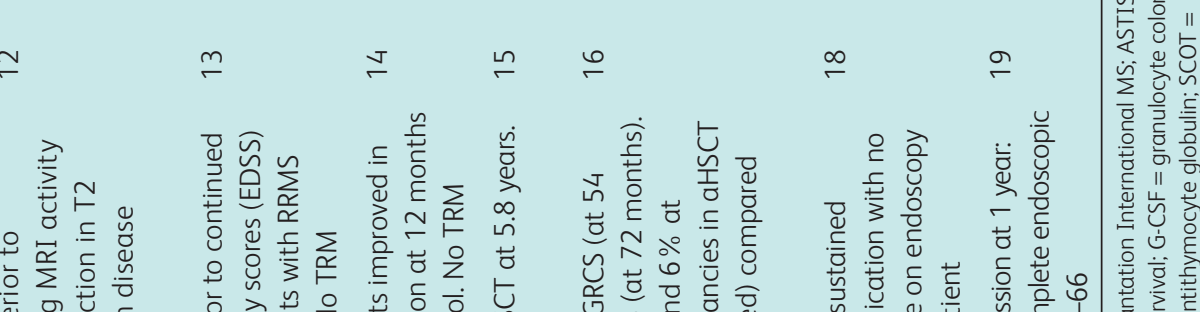

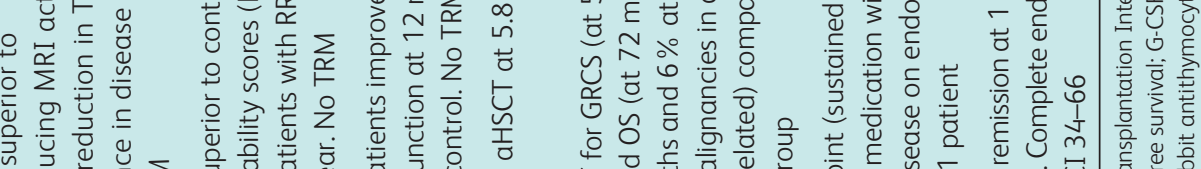

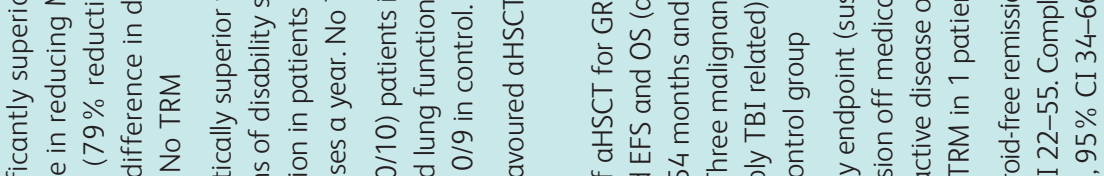

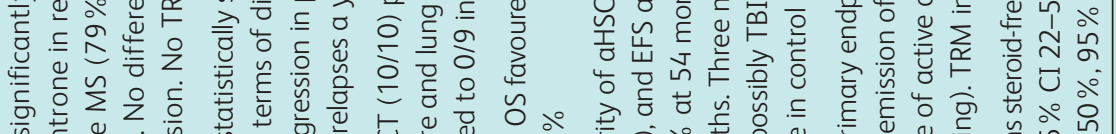

흥를

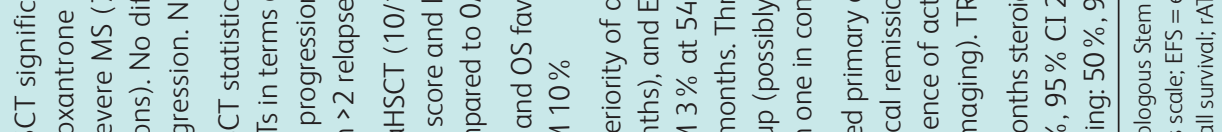

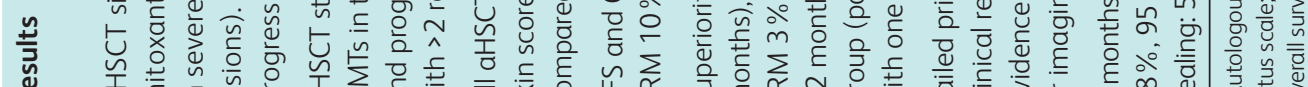

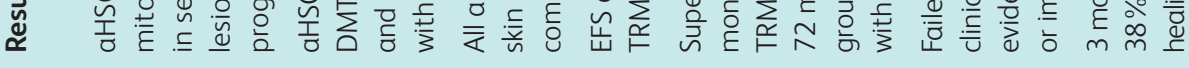

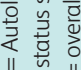

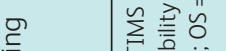

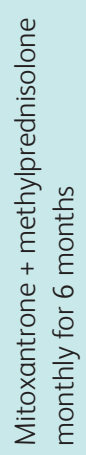

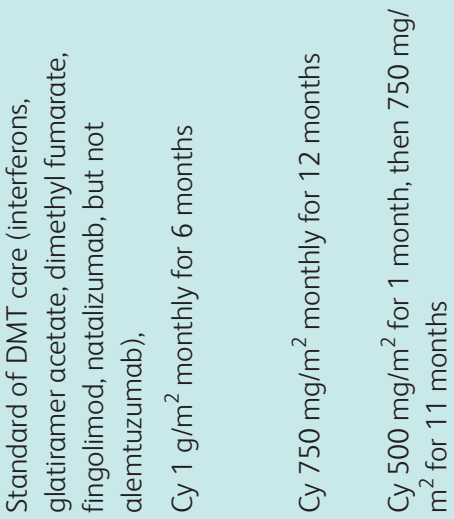

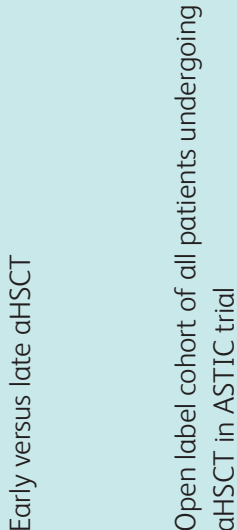

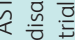

产

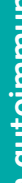

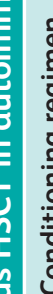

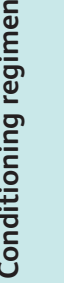

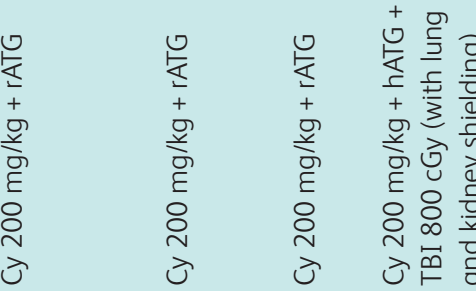

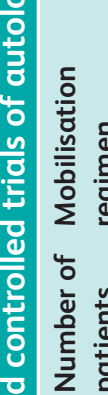
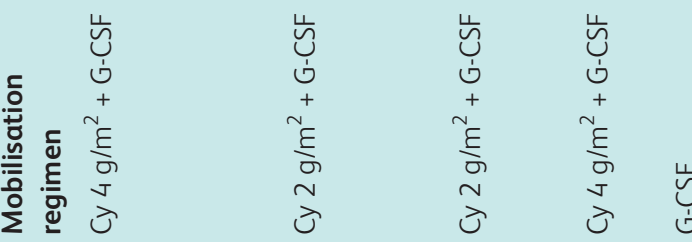

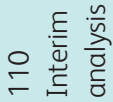

을 焉宅

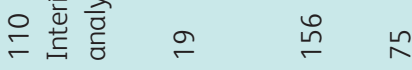

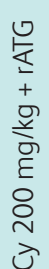

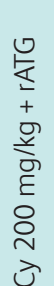

要

品 $\overline{0}^{\circ}$,

등 ॥

잉

蒂

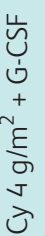

西 $\quad \sum_{i=}^{n}$

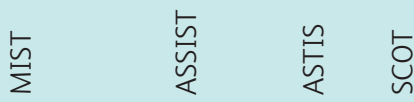

นุ

$\infty$

ํㅣㄹ

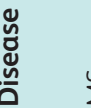

$\sum$

$\breve{\sim}$

$\tilde{\sim} \backsim$

४

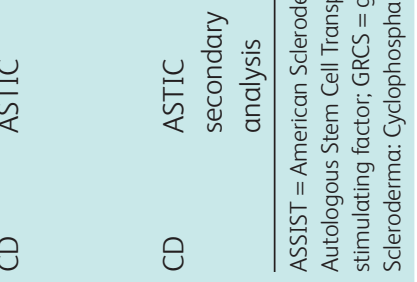


patients who relapsed after aHSCT subsequently responded to reintroduction of anti-TNF therapy. ${ }^{6,20}$

Further research is required to assess the place of aHSCT in the management of patients with treatment refractory and poor prognosis CD. ASTIClite is an NIHR-funded randomised controlled trial that will assess the benefit and risks of a low intensity mobilisation and conditioning HSCT regimen compared to the best currently available medical care in patients with significant $\mathrm{CD}$ refractory to at least two classes of biologic therapy. The primary endpoint will be mucosal healing, and patients who are randomised to HSCT but have disease recurrence at 6 months will restart anti-TNF therapy. ${ }^{21}$

\section{Other diseases}

A variety of other autoimmune diseases have been treated with aHSCT and are summarised in Table 2. In these rarer disease indications, it has been challenging to conduct large-scale clinical trials, at least of the randomised type, and this is where the transplant registries have been especially useful. Outcomes of HSCT in these indications can be accessed via recent reviews of the field. ${ }^{3,4}$

\section{Mechanisms of action}

In the early stages the administration of high doses of cytotoxic therapy has a profound anti-inflammatory effect (see Fig 1, under 'aHSCT ablation'). Subsequently, post-transplant immune reconstitution studies have supported re-diversification of T-cell repertoire, with quantitative and qualitative changes in T-regulatory cell numbers (see Fig 1, under 'aHSCT reconstitution'). Other immune compartments, such as naive B cells and NK cells may also be regenerated. Effects on the adaptive immune system persist well beyond the transplant period and the lymphocyte repopulation phase, supporting the notion of an immune reset or reboot. In addition to providing insights on immune reconstitution, concurrent scientific studies conducted while destroying and rebuilding dysfunctional immune systems may also provide useful insights into the aetiology and pathogenesis of autoimmune diseases. ${ }^{7,11,21}$

\section{Conclusions}

Autologous HSCT has been used in a wide range of AD clinical settings but the field has focused on three areas in which disease control remains unsatisfactory despite the advent of biologicals and other modern immunomodulatory therapies: MS, diffuse cutaneous SSC and CD. There may be health economic benefits from a single one-off procedure versus years of ongoing biological treatment. Even so, treatment with aHSCT is intensive with a range of significant risks and toxicities, which, despite being routine to transplant haematologists, have variable acceptability to disease specialists. Close collaboration between transplant haematologists and relevant disease specialists is essential in patient selection, clinical management and follow-up. Early referral of patients, where there is good general fitness as well as the potential of tissue damage reversibility is essential to achieve the best clinical outcomes while minimising the risks of toxicity. Ideally, patients should be treated on a clinical trial or at least their data submitted to a transplant registry. Concurrent scientific studies will help elucidate the mechanism of action while shedding light on the underlying pathogenesis of ADs.

\section{Conflicts of interest}

JAS declares honoraria from Sanofi and Jazz. PAM declares travel support and speaker honoraria from Bayer HealthCare, Bayer Pharma, Biogen Idec, Merck-Serono and Sanofi Aventis. AL declares advisory
Disease susceptibility

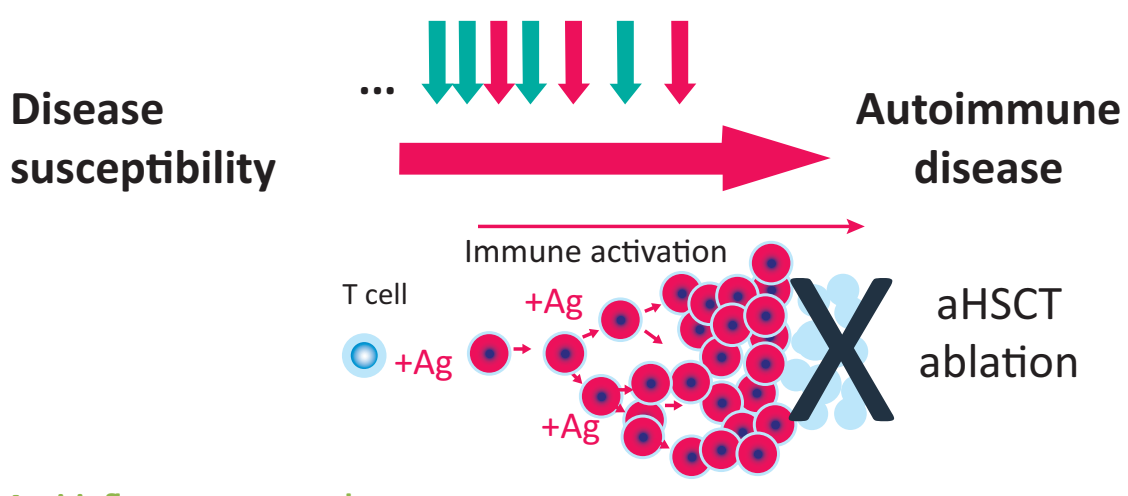

Environmental hits

- Pathogen and commensal microbes eg viruses, microbiome

- Other environmental factors eg smoke, vitamin $\mathrm{D}$ deficiency

Anti-inflammatory regulators

$>$ CD4+/CD25high/FoxP3 Tregs

NK regs

CD8+/CD28-/CD57+ T cells

CTLA-4 expression

PD-1 expression

TGF- $\beta 1$ secretion

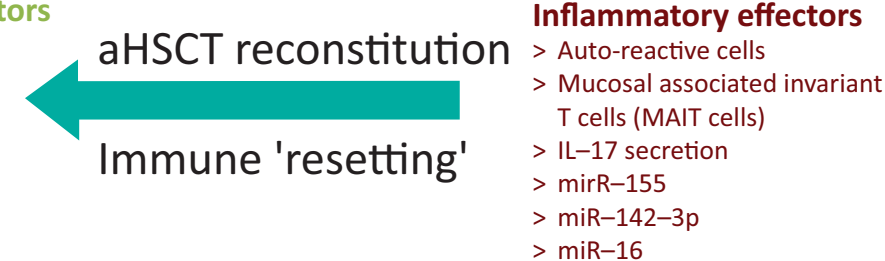

Fig 1. Potential mechanisms of action of aHSCT in autoimmune diseases. In individuals with genetic susceptibility towards autoimmune disease, exposure to multiple environmental factors such as viral infection and toxic or metabolic damage (top), may induce autoimmune disease through aberrant antigen (Ag) mediated activation of $\mathrm{T}$ cells (centre). In aHSCT the cytotoxic chemotherapy ablates the lymphoid system including $T$ lymphocyte immunity (as depicted). The leukocyte depletion enables an immune reconstitution that restores the predominance of anti-inflammatory regulating factors (bottom left) over inflammatory effectors (bottom right). These mechanisms may explain induction of long-lasting suppression of some autoimmune diseases by aHSCT . 
board membership, speaker fees or consultancy for MSD, Abbvie, Janssen, Takeda, Medtronic, Vifor Pharma and Shield Therapeutics. There are no other declarations.

\section{Acknowledgements}

We thank Manuela Badoglio in the EBMT Paris Office for an update of the EBMT Registry for autoimmune diseases. The ASTIClite project is funded by the Efficacy and Mechanism Evaluation (EME*) Programme, an MRC and NIHR partnership. Disclaimer: The views expressed in this publication are those of the author(s) and not necessarily those of the MRC, NHS, NIHR or the Department of Health and Social Care.

\section{References}

1 Passweg JR, Baldomero $\mathrm{H}$, Bader $\mathrm{P}$ et al. Is the use of unrelated donor transplantation leveling off in Europe? The 2016 European Society for Blood and Marrow Transplant activity survey report. Bone Marrow Transplant 2018 doi: 10.1038/s41409-018-0153-1.

2 Snowden JA, Badoglio M, Labopin $\mathrm{M}$ et al. Evolution, trends, outcomes, and economics of hematopoietic stem cell transplantation in severe autoimmune diseases. Blood Adv 2017;1:2742-55.

3 Kelsey PJ, Oliveira MC, Badoglio M et al. Haematopoietic stem cell transplantation in autoimmune diseases: From basic science to clinical practice. Curr Res Transl Med 2016;64:71-82.

4 Snowden JA, Saccardi R, Allez M et al. Haematopoietic SCT in severe autoimmune diseases: updated guidelines of the European Group for Blood and Marrow Transplantation. Bone Marrow Transplant 2012;47:770-90.

5 Farge D, Burt RK, Oliveira MC et al. Cardiopulmonary assessment of patients with systemic sclerosis for hematopoietic stem cell transplantation: recommendations from the European Society for Blood and Marrow Transplantation Autoimmune Diseases Working Party and collaborating partners. Bone Marrow Transplant 2017; 52:1495-503.

6 Snowden JA, Panes J, Alexander T et al. Autologous haematopoietic stem cell transplantation (AHSCT) in severe Crohn's Disease: a review on behalf of ECCO and EBMT. J Crohns Colitis 2018;12:476-88.

7 Alexander T, Bondanza A, Muraro PA et al. SCT for severe autoimmune diseases: consensus guidelines of the European Society for Blood and Marrow Transplantation for immune monitoring and biobanking. Bone Marrow Transplant 2015;50:173-80.

8 Kowal-Bielecka O, Fransen ], Avouac ] et al. EUSTAR Coauthors. Update of EULAR recommendations for the treatment of systemic sclerosis. Ann Rheum Dis 2017:76:1327-39.

9 Bell SM, Sharrack B, Snowden JA. Autologous hematopoietic cell transplantation in multiple sclerosis. Expert Opin Biol Ther 2017;17:77-86.

*The EME Programme is funded by the MRC and NIHR, with contributions from the CSO in Scotland and Health and Care Research Wales and the HSC R\&D Division, Public Health Agency in Northern Ireland.
10 Muraro PA, Pasquini M, Atkins HL et al. Long-term outcomes after autologous hematopoietic stem cell transplantation for multiple sclerosis. JAMA Neurol 2017;74:459-69.

11 Muraro PA, Martin R, Mancardi GL et al. Autologous haematopoietic stem cell transplantation for treatment of multiple sclerosis. Nat Rev Neurol 2017:13:391-405.

12 Mancardi GL, Sormani MP, Gualandi F et al. Autologous hematopoietic stem cell transplantation in multiple sclerosis: a phase II trial. Neurology 2015;84:981-8

13 Burt RK, Balabanov R, Snowden JA et al. Non-myeloablative hematopoietic stem cell transplantation (HSCT) is superior to disease modifying drug (DMD) treatment in highly active relapsing remitting multiple sclerosis (RRMS): interim results of the Multiple Sclerosis International Stem cell Transplant (MIST) randomized trial. Neurology 2018;90(Suppl 15):S36.004.

14 Burt RK, Shah SJ, Dill K et al. Autologous non-myeloablative haemopoietic stem-cell transplantation compared with pulse cyclophosphamide once per month for systemic sclerosis (ASSIST): an open-label, randomised phase 2 trial. Lancet 2011;378:498506.

15 van Laar JM, Farge D, Sont JK et al. Autologous hematopoietic stem cell transplantation vs intravenous pulse cyclophosphamide in diffuse cutaneous systemic sclerosis: a randomized clinical trial. JAMA 2014:311:2490-8.

16 Sullivan KM, Goldmuntz EA, Keyes-Elstein L et al. Myeloablative autologous stem-cell transplantation for severe scleroderma. $N$ Engl J Med 2018;378:35-47.

17 Alexander T, Hiepe F. Autologous haematopoietic stem cell transplantation for systemic lupus erythematosus: time ready for a paradigm shift? Clin Exp Rheumatol 2017;35:359-61.

18 Hawkey C], Allez M, Clark MM et al. Autologous hematopoetic stem cell transplantation for refractory Crohn disease: a randomized clinical trial. JAMA 2015;314:2524-34.

19 Lindsay JO, Allez M, Clark M et al. Autologous stem-cell transplantation in treatment-refractory Crohn's disease: an analysis of pooled data from the ASTIC trial. Lancet Gastroenterol Hepatol 2017:2:399-406.

20 Brierley CK, Castilla-Llorente C, Labopin M et al. A retrospective survey of long-term outcomes from the European Society for Blood and Marrow Transplantation. J Crohns Colitis 2018 doi: 10.1093/ ecco-jcc/jjy069.

21 Pockley G, Lindsay JO, Foulds GA et al. immune reconstitution after autologous haematopoietic stem cell transplantation in Crohn's disease: current status and future directions. A review on behalf of the EBMT Autoimmune Diseases Working Party and the ASTIClite Study Investigators. Front Immunol 2018;9:646.

Address for correspondence: Professor John Snowden, Sheffield Teaching Hospitals NHS Foundation Trust, Haematology, Royal Hallamshire Hospital, Sheffield S10 2JF, UK.

Email: john.snowden@sth.nhs.uk 